\title{
What can pre-solar grains tell us about the solar nebula?
}

\author{
Gary R. Huss ${ }^{1}$ and Bruce T. Draine ${ }^{2}$ \\ ${ }^{1}$ Hawai'i Institute of Geophysics and Planetology, University of Hawai'i at Mānoa, \\ 1680 East-West Road, Honolulu, HI 96822, USA \\ email: ghuss@higp.hawaii.edu \\ ${ }^{2}$ Department of Astrophysical Sciences, Princeton University, \\ 108 Peyton Hall, Princeton, NJ 08544-1001, USA \\ email:draine@astro.princeton.edu
}

\begin{abstract}
Several types of pre-solar grains, grains that existed prior to solar system formation, have been found in the fine-grained components of primitive meteorites, interplanetary dust particles (IDPs), and comet samples. Known pre-solar components have isotopic compositions that reflect formation from the ejecta of evolved stars. Other pre-solar materials may have isotopic compositions very similar to solar system materials, making their identification as presolar grains problematic. Pre-solar materials exhibit a range of chemical and thermal resistance, so their relative abundances can be used to probe the conditions in the solar nebula. Detailed information on the relative abundances of pre-solar and solar-system materials can provide information on the temperatures, radiation environment, and degree of radial mixing in the early solar system.
\end{abstract}

Keywords. pre-solar grains, solar system: formation, ISM: abundances, ISM: dust

\section{Introduction}

Pre-solar grains are solid objects that existed in interstellar space prior to the formation of the solar system and have survived within meteorites and comets until the present day to be studied in the laboratory. The study of pre-solar grains has been mainly the study of isotopic anomalies, which have historically been defined as isotopic compositions that could not have been produced from the solar system composition, as measured in terrestrial and lunar samples and meteorites, by known processes operating within the solar system. The first anomalies of this type were found in noble gases from primitive meteorites (e.g., Reynolds \& Turner 1964; Black \& Pepin 1969), although these anomalies were not initially thought of as signatures of pre-solar materials. Additional discoveries of isotopic anomalies in oxygen (Clayton et al. 1973; Clayton et al. 1977), hydrogen (e.g., Yang \& Epstein 1984), carbon (e.g., Swart et al. 1983), titanium (e.g., Niederer et al. 1980), and other elements eventually led to a consensus that pre-solar materials were not completely homogenized when the solar system formed. The first pre-solar mineral grains to be identified were found after a decades-long search for the carriers of anomalous noble-gas components. They were diamond (carrier of Xe-HL; Lewis et al. 1987), silicon carbide (Ne-E(H) and Xe-S; Tang \& Anders 1988) and graphite (Ne-E(L); Amari et al. 1990). Since these initial identifications, $\sim 20$ pre-solar compounds have been identified in meteorites and interplanetary dust particles (Table 1). However, as the abundances clearly show, we have not yet identified a majority of the material that made up the Sun's parent molecular cloud.

Pre-solar materials can be divided into two types, circumstellar condensates, which formed from the ejecta of dying stars and carry the isotopic signature of nucleosynthesis 
Table 1. Types and properties of pre-solar materials identified in meteorites and IDPs

\begin{tabular}{|c|c|c|c|c|c|}
\hline Material & Source & $\begin{array}{l}\text { Grain Size } \\
\qquad(\mu \mathrm{m})\end{array}$ & $\begin{array}{l}\text { Abundance } \\
(\mathrm{ppm}) \dagger\end{array}$ & $\begin{array}{l}\text { Chemical } \\
\text { resistance }\end{array}$ & $\begin{array}{l}\text { Thermal } \\
\text { resistance }\end{array}$ \\
\hline Diamond & & $\sim 0.002$ & $\sim 1400$ & & \\
\hline P3 fraction & $?$ & & & high & low \\
\hline HL fraction & circumstellar & & & very high & high \\
\hline Silicon carbide & circumstellar & $0.1-20$ & $13-14$ & high & high \\
\hline Graphite & circumstellar & $0.1-10$ & $7-10$ & moderate & low \\
\hline D-rich organics & interstellar & & & low to mod. & low to mod. \\
\hline P1 noble gas carrier & interstellar & $*$ & $*$ & moderate & high \\
\hline Corundum $\left(\mathrm{Al}_{2} \mathrm{O}_{3}\right)$ & circumstellar & $0.5-3$ & 0.01 & high & very high \\
\hline Spinel $\left(\mathrm{MgAl}_{2} \mathrm{O}_{4}\right)$ & circumstellar & $0.1-3$ & 1.2 & high & very high \\
\hline Hibonite $\left(\mathrm{CaAl}_{12} \mathrm{O}_{19}\right)$ & circumstellar & $1-2$ & 0.02 & high & very high \\
\hline $\left.\begin{array}{l}\text { Forsterite }\left(\mathrm{Mg}_{2} \mathrm{SiO}_{4}\right) \\
\text { Enstatite }\left(\mathrm{MgSiO}_{3}\right)\end{array}\right\}$ & circumstellar & $0.2-0.5$ & $10-1800$ & low to mod. & high \\
\hline Amorphous silicates & circumstellar & $0.2-0.5$ & $20-3600$ & low & moderate \\
\hline
\end{tabular}

Notes.

Other pre-solar materials include $\mathrm{TiC}, \mathrm{MoC}, \mathrm{ZrC}, \mathrm{RuC}, \mathrm{FeC}, \mathrm{Si}_{3} \mathrm{~N}_{4}, \mathrm{TiO}_{2}$, and Fe-Ni metal. $\dagger$ Abundance in fine-grained fraction (= matrix in primitive chondrites).

$*{ }^{132} \mathrm{Xe}-\mathrm{P} 1$ content in carbonaceous residue from primitive chondrites is $\sim(1.2-1.4) \times 10^{-8} \mathrm{cc}-$ $\mathrm{STP} /$ gram, but the carrier itself is still unknown.

Data: Huss \& Lewis (1995); Huss et al. (1996); Huss et al. (2003); Messenger et al. (2006).

in the parent star, and interstellar material, which grew from the gas phase in interstellar space, primarily in molecular clouds. Most pre-solar materials identified in the laboratory are circumstellar condensates, which carry the largest isotopic anomalies relative to solar system materials. Astronomical observations indicate that AGB stars are prolific dust producers. Most of the pre-solar grains studied to date are from AGB stars, although we note that grains below $\sim 0.3 \mu \mathrm{m}$ are currently almost impossible to study. Amorphous silicates, Mg-rich crystalline silicates, $\mathrm{Al}_{2} \mathrm{O}_{3}$, and FeMg oxides are observed in the circumstellar dust shells of O-rich AGB stars (Molster et al. 2002; Tielens et al. 2005). Silicon carbide is observed in C-rich dust from AGB stars (Speck et al. 1997). Amorphous silicates and amorphous $\mathrm{C}$ have been reported from observations of the interstellar medium (see, e.g., review by Draine (2003) and references therein). In dark molecular clouds, the grains acquire mantles consisting primarily of $\mathrm{H}_{2} \mathrm{O}$ ice but often containing appreciable fractions of $\mathrm{CO}, \mathrm{CO}_{2}, \mathrm{CH}_{3} \mathrm{OH}$, and other species.

\section{Differential processing of pre-solar materials in the solar nebula}

Pre-solar materials exhibit a wide range of resistance to heating and chemical processing (Table1). This opens the possibility that their relative abundances can be used as probes of conditions in the solar nebula. For example, if a portion of a molecular cloud containing all of the materials in Table 1 (plus many others) is heated to $1000 \mathrm{~K}$, we would expect that the P3 fraction of diamond, graphite, D-rich organics, and some of the poorly crystalline silicates would be destroyed, either through vaporization or through chemical reaction. At higher temperatures, other components would also be destroyed. Heating in a highly oxidizing environment would affect carbonaceous and organic components more than oxides, while reducing environments would increase the survival of carbonaceous and organic components. An initial study of unmetamorphosed chondrites comparing abundances of diamond, silicon-carbide, and graphite in the matrices with the bulk compositions of the meteorites shows a correlation between depletions of fragile 
pre-solar components and depletions of volatile and moderately volatile elements (Huss et al. 2003). This correlation suggests that the pre-solar grains track the thermal processing in the solar nebula that produced the bulk compositions of different classes of meteorites from molecular-cloud material.

\section{Formation of organic compounds}

Isotopic and chemical compositions of organic materials in meteorites, IDPs, and comet samples may also provide information about conditions in the solar nebula. For example, dense molecular clouds contain a wide variety of relatively complex organic molecules synthesized by radiation-driven chemistry in the gas phase and in icy grain mantles (e.g., Allamandola et al. 1988). Some of these molecules, such as those containing the $\mathrm{OCN}^{-}$ion, are more abundant in the spectra of protostars than in the spectra of background stars (Pendleton et al. 1999), suggesting that radiation-driven chemistry may be enhanced in the immediate vicinity of star-formation. Many molecules synthesized in laboratory experiments simulating conditions in molecular clouds are similar or identical to compounds found in primitive meteorites (Bernstein et al. 2001). There are clear isotopic signatures (e.g., high $\mathrm{D} / \mathrm{H}$ ) associated with organic molecules produced by radiation chemistry in cold molecular clouds and in dense, cold regions of an accretion disk (e.g., Sandford et al. 2001). Interstellar grains may be heavily deuterated in diffuse regions (Linsky et al. 2006), with D presumably present in hydrocarbons, possibly including PAHs (Draine 2006). Detailed work on organics in meteorites and interplanetary dust particles is just beginning, as new tools become available. Identifying specific molecules that carry extreme isotope anomalies will help identify the chemical pathways of their creation, which in turn will constrain the environment of formation, either in the molecular cloud or in the early solar nebula.

\section{Circulation and mixing in the solar nebula}

An a priori knowledge of the types of materials in the Sun's parent molecular cloud provides the basis for which to track circulation and mixing in the solar nebula. Late-stage stars produce both amorphous and crystalline silicates in their outflows. The proportion of crystalline silicates may be anywhere from 5-10\% up to as much as $40 \%$, but detection is difficult (e.g., Molster et al. 2002; Kemper et al. 1999). However, crystalline silicates comprise $<2 \%$ of the silicate material in interstellar space (Kemper et al. 2005). Crystalline silicates can be destroyed by interstellar shocks and amorphized by cosmic rays, thus reducing their abundance. On the other hand, observations of young stellar systems show that the abundance of crystalline silicates is much higher in the inner disk than in the outer disk, but that even the outer disks show more crystalline silicates than the interstellar medium (Tielens et al. 2005). These observations indicate that crystalline silicates are produced in the inner disk and are mixed outward.

Chemical thermodynamics predicts that pure Mg-end-member olivine (forsterite) and pyroxene (enstatite) grains will be the first major silicate phases to condense from a cooling gas of stellar or solar composition (Grossman 1972; Lattimer et al. 1978). In contrast, olivine and pyroxene that crystallize from a melt typically contain Fe and other elements. Nearly pure Mg-end-member forsterite and enstatite are observed along with amorphous silicates and Fe-bearing olivine in the matrices of the most primitive chondrites (e.g., Brearley 1993). Observations of dust released by comets and of porous chondritic IDPs thought to have come from comets also show both crystalline and amorphous silicates (e.g., Wooden et al. 2004; Keller \& Messenger 2005). These data sets suggest that 
mixing of high-temperature nebular components from the inner to the outer solar system was significant. Transport of high-temperature material to the outer solar system may have occurred via turbulent mixing (Cuzzi et al. 2003), by X-winds (Shu et al. 1996), or by other unknown processes. Detailed studies of pre-solar and high-temperature nebula components in primitive meteorites, IDPs, and comet samples will be critical in interpreting the astronomical observations and in understanding mixing processes in the early solar nebula.

\section{Acknowledgements}

Supported by NASA grant NNG05GG48G (GRH) and NSF grant AST-0406883 (BTD).

\section{References}

Allamandola, L. J., Sandford, S. A., \& Valero, G. 1988, Icarus, 76, 225

Amari, S., Anders, E., Virag, A., \& Zinner, E. 1990, Nature, 345, 238

Bernstein, M. P., Dworkin, J. P., Sandford, S. A., \& Allamandola, L. J. 2001, Meteorit. Planet. Sci., 36, 351

Black, D. C., \& Pepin, R. O. 1969, Earth Planet. Sci. Lett., 6, 395

Brearley, A. J. 1993, Geochim. Cosmochim. Acta, 57, 1521

Clayton, R. N., Grossman, L., \& Mayeda, T. K. 1973, Science, 182, 485

Clayton, R. N., Onuma, N., Grossman, L., \& Mayeda, T. K. 1977, Earth Planet. Sci. Lett., 34, 209

Cuzzi, J. N., Davis, S. S., \& Dobrovolskis, A. R. 2003, Icarus, 166, 385

Draine, B. T. 2003, ARAA, 41, 241

Draine, B. T. 2006, in: G. Sonneborn, H. W. Moos, \& B.G. Andersson (eds.), Astrophysics in the Far Ultraviolet, ASP-CS 348, 58

Grossman, L. 1972, Geochim. Cosmochim. Acta, 36, 597

Huss, G. R., \& Lewis, R. S. 1995, Geochim. Cosmochim. Acta, 59, 115

Huss, G. R., Lewis, R. S., \& Hemkin, S. 1996, Geochim. Cosmochim. Acta, 60, 3311

Huss, G. R., Meshik, A. P., Smith, J. B., \& Hohenberg, C. M. 2003, Geochim. Cosmochim. Acta, 67, 4823

Keller, L. P., \& Messenger, S. 2005, in: A. N. Krot, E. R. D. Scott \& B. Reipurth (eds.), Chondrites and the Protoplanetary Disk, ASP-CS 341, 657

Kemper, F., Spaans, M., Jansen, D. J., Hogerheijde, M. R., van Dishoeck, E. F., \& Tielens, A. G. G. M. $1999, A p J, 515,649$

Kemper, F., Vriend, W. J., \& Tielens A. G. G. M. 2005, ApJ, 633, 534

Lattimer, J. M., Schramm, D. N., \& Grossman, L. 1978, ApJ, 219, 230

Lewis, R. S., Tang, M., Wacker, J. F., Anders, E., \& Steele, E. 1987, Nature, 326, 160

Linsky, J. L., Draine, B. T., Moos, H. W., et al. 2006, ApJ, 647, 1106

Messenger, S., Sandford, S., \& Brownlee, D. 2006, in: D. S. Lauretta \& H. Y. McSween (eds.), Meteorites and the Early Solar System II (Univ. of Arizona), p. 187

Molster, F. J., Waters L. B. F. M., Tielens, A. G. G. M., et al. 2002, A\& A, 382, 241

Niederer, F. R., Papanastassiou, D. A., \& Wasserburg, G. J. 1980, ApJ (Letters), 240, L73

Pendleton, Y. J., Tielens, A. G. G. M., Tokunaga, A. T., \& Bernstein, M. P. 1999, ApJ, 513, 294

Reynolds, J. H., \& Turner, G. 1964, J. Geophys. Res. 69, 3263

Sanford, S. A., Bernstein, M. P., \& Dworkin, J. P. 2001, Meteorit. Planet. Sci. 36, 1117

Shu, F. H., Shang, H., \& Lee, T. 1996, Science, 271, 1545

Speck, A. K., Barlow, M. J., \& Skinner, C. J. 1997, MNRAS, 288, 431

Swart, P. K., Grady M. M., Pillinger C. T., Lewis R. S., \& Anders E. 1983, Science, 220, 406

Tang, M., \& Anders, E. 1988, Geochim. Cosmochim. Acta, 52, 1235

Tielens, A. G. G. M., Waters, L. B. F. M., \& Bernatowicz, T. J. 2005, in: A. N. Krot, E. R. D. Scott, \& B. Reipurth (eds.), Chondrites and the Protoplanetary Disk, ASP-CS, 341, 605

Wooden, D. H., Woodward, C. E., \& Harker D. E. 2004, ApJ (Letters), 612, L77

Yang, J., \& Epstein S. 1984, Nature, 311, 544 